\title{
Electrochemical single-molecule conductivity of duplex and quadruplex DNA
}

\author{
Zhang, Ling; Zhang, Jingdong; Ulstrup, Jens
}

Published in:

Current Opinion in Electrochemistry

Link to article, DOI:

10.1016/j.coelec.2017.09.005

Publication date:

2017

Document Version

Peer reviewed version

Link back to DTU Orbit

Citation (APA):

Zhang, L., Zhang, J., \& Ulstrup, J. (2017). Electrochemical single-molecule conductivity of duplex and quadruplex DNA. Current Opinion in Electrochemistry, 7, 66-74. https://doi.org/10.1016/j.coelec.2017.09.005

\section{General rights}

Copyright and moral rights for the publications made accessible in the public portal are retained by the authors and/or other copyright owners and it is a condition of accessing publications that users recognise and abide by the legal requirements associated with these rights.

- Users may download and print one copy of any publication from the public portal for the purpose of private study or research.

- You may not further distribute the material or use it for any profit-making activity or commercial gain

- You may freely distribute the URL identifying the publication in the public portal

If you believe that this document breaches copyright please contact us providing details, and we will remove access to the work immediately and investigate your claim. 


\section{Highlights}

- $\quad$ Single-molecule electrochemistry of duplex and G-quadruplex oligonucleotides (OGNs)

- Electrochemical in situ STM of pure and redox marked duplex and G-quadruplex OGNs

- Iron protoporphyrin IX labeled 15-base 12G-quadruplex (DNAzyme) at single-crystal electrodes

- Long-range electrochemical electron transfer of redox labelled duplex OGNs and DNAzyme

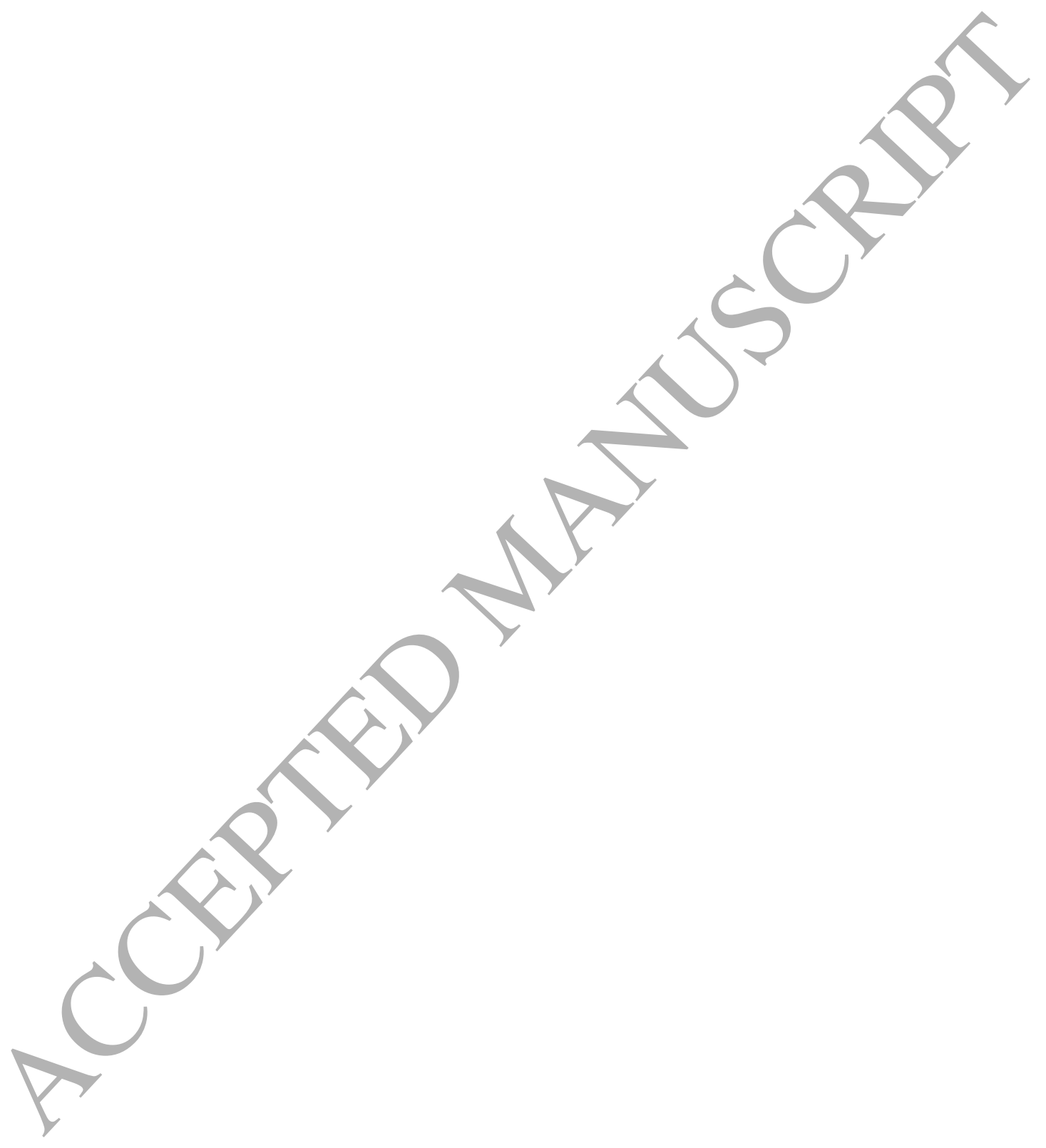


Electrochemical Single-molecule Conductivity of Duplex and Quadruplex DNA

\section{Ling Zhang, Jingdong Zhang,* and Jens Ulstrup*}

Department of Chemistry, Building 207, Technical University of Denmark, DK-2800 Kgs. Lyngby, Denmark

*Corresponding authors:

Jingdong Zhang jz@kemi.dtu.dk Jens Ulstrup ju@kemi.dtu.dk

\section{Highlights}

- Single-molecule electrochemistry of duplex and G-quadruplex oligonucleotides (OGNs)

- Electrochemical in situ STM of pure and redox marked duplex and G-quadruplex OGNs

- Iron protoporphyrin IX labeled 15-base 12G-quadruplex (DNAzyme) at single-crystal electrodes

- Long-range electrochemical electron transfer of redox labelled duplex OGNs and DNAzyme 
Photoinduced and electrochemical charge transport in DNA (oligonucleotides, OGNs) and the notions "hopping", superexchange, polaron, and vibrationally gated charge transport have been in focus over more than two decades. In recent years mapping of electrochemical charge transport of pure and redox marked single- and double-strand OGNs has reached the single-molecule level based i.a. on electrochemical in situ scanning tunnelling microscopy (STM) and break-junction (B-J) STM. There are much fewer such reports on "non-canonical" OGN structures such as G-quadruplexes. We discuss first single-molecule electrochemical conductivity of pure and redox marked duplex OGNs, and address next electrochemistry and electrochemical conductivity in the few reported monolayer and single-molecule G-quadruplex studies. Facile electrochemical electron transfer of iron protoporphyrin IX stacked onto three-quartet 12-guanine quadruplex ("DNAzyme") and in situ highresolution molecular structures are particularly noted.

\section{Introduction}

Experimental and theoretical studies of electron or hole transfer, ET/HT of single (bio)molecules such as proteins ${ }^{1-9}$ and $\mathrm{DNA}^{10-16}$ have reached high levels of sophistication. The ways single redox metalloprotein molecules operate are increasingly well understood, e.g. from bioelectrochemistry and electrochemical scanning tunnelling microscopy/spectroscopy (in situ STM/STS) directly in aqueous biological media. DNA (oligonucleotides, OGN) conductivity offers other perspectives for oxidative damage repair ${ }^{17-19}$ and molecular "devices" 16,20-24. Binding of organic redox molecules or transition metal complexes are core notions in long-range ET through the double strand ${ }^{17-19,25-28}$, extended now to PNA, treated elsewhere in this Current Opinion issue, and G-quadruplexes addressed presently.

DNA conductivity rests broadly on: (1) photo-excitation, followed by hole transport along the OGN strand $^{17-19,21-24}$; (2) electrochemical ET of a chelator "through" surface bound $\mathrm{OGN}^{26-28}$; (3) singlemolecule imaging and conductivity by STM and B-J STM ${ }^{1,10,11,15}$. "Hopping" and "superexchange" (tunnelling) are two limiting mechanisms ${ }^{20,21}$, with polaron conductivity ${ }^{29}$ and ET/HT via dynamically populated intermediate states $s^{30-34}$ as other notions. Hopping involves sequential, vibrationally relaxed ET between base pairs or base pair clusters, Fig.1. The generic rate constants are ${ }^{20,33}$

$W_{\text {hop }}=\kappa \frac{\omega_{\text {eff }}}{2 \pi} \exp \left(-\frac{\left[\lambda+\Delta G^{0}\right]^{2}}{4 \lambda k_{B} T}\right) ; \kappa=\left(T_{D A}\right)^{2} \sqrt{\frac{4 \pi^{3}}{\lambda \hbar^{2} \omega_{e f f}^{2} k_{B} T}}$, if $\kappa<1$, otherwise $\kappa=1$

$\lambda$ is the reorganization free energy, $\Delta G^{0}$ the reaction free energy, $\omega_{\text {eff }}$ the effective vibrational frequency of the ET hop, $k_{\mathrm{B}}$ Boltzmann's constant and $T$ the temperature. $T_{D A}$ in the electronic transmission coefficient $\mathcal{c}$ couples the donor (D) and acceptor (A) states, commonly dominated by guanine-cytosine (GC) pairs, via intermediate adenine-thymine (AT) tunnelling "matter"20,21. A coarse-grained distance $(R)$ dependent $T_{D A}$ form for ET/HT through $M$ intermediate states is

$$
T_{D A} \approx \frac{\beta_{D} \beta_{A}}{\gamma}\left(\frac{\gamma}{\Delta \varepsilon}\right)^{M} \rightarrow T_{D A}=\left(T_{D A}^{0}\right) \exp \left(-\beta_{\text {eff }} R\right) ; \quad \beta_{\text {eff }}=\frac{1}{a} \ln \frac{\Delta \varepsilon}{\gamma}
$$

where $\Delta \varepsilon$ is the energy gap between the donor and nearest bridge group levels, $\beta_{\mathrm{D}}$ and $\beta_{\mathrm{A}}$ coupling of the donor and acceptor to their nearest bridge groups, and $\gamma$ the electronic coupling between neighbouring bridge groups of structural extension $a$. Large $\Delta \varepsilon$ and small $\gamma$ thus give fast distance fall-off. "Superexchange", eq.(2), means that ET/HT is transmitted via high-energy AT base pairs, without physical charge localization but by efficient off-resonance nearest neighbour coupling. Extensions of eqs.(1) and (2) have become a frame for multiphonon in situ STM processes and current/overpotential/bias voltage STS of single redox (bio)molecules ${ }^{2,31,32,-35}$. 


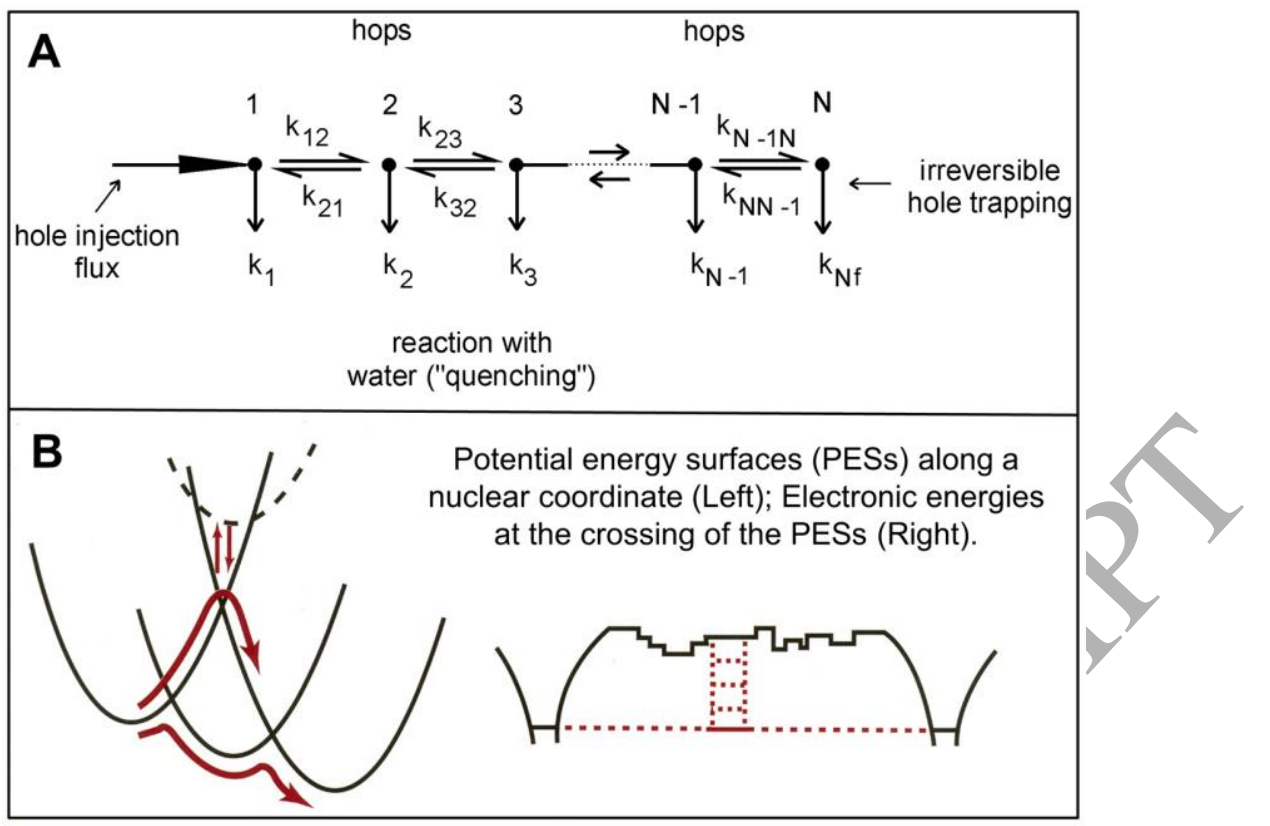

Fig. 1 Two schematic views of long-range ET/HT through OGN molecular strands. Details in text.

The two limiting mechanisms are illustrated in Fig.1. Fig.1A illustrates photo-induced ET/HT and random "hopping" of the excess charge along the strand, interrupted by "quenching" $\left(k_{1} \ldots k_{\mathrm{Nf}}\right)$ and eventual trapping. Fig.1B, left shows corresponding potential (free) energy surfaces spanned by a nuclear coordinate to show differences between "hopping" and superexchange via a single intermediate electronic state. "Hopping" via a temporarily populated low-lying intermediate state is represented by the fully drawn intermediate state potential surface. This state may or may not relax vibrationally prior to the subsequent ET/HT step. In "superexchange" a high-energy intermediate state (drawn by the dashed line) mediates ET/HT purely electronically, without physical electronic population. Fig.1B, right shows a corresponding electronic energy diagram spanned by the spatial coordinate(s) of the transferring electron (hole). The Coulomb-like potential wells are at resonance between the donor and acceptor states at the crossing of the initial and final potential surfaces. The potential wells drawn in dashed burgundy lines illustrate intermediate electronic energies from superexchange (high energy) to "hopping" with resonance between the three states.

Hopping has emerged as a consistent mechanism for photo-induced long-range, weakly distance dependent ET/HT $(>200 \mathrm{~nm})^{17-24}$ with $\tau_{c s}^{N} \approx\left(W_{h o p}\right)^{-1} N^{\eta}$ where $\tau_{c s}^{N}$ is the charge separation time, $N$ the number of hops, and $\eta \approx 1-2$ a numerical factor ${ }^{20,21}$. Electrochemical "hopping" is less obvious, as this involves electrochemical oxidation of DNA bases. Such signals have eluded identification at gold substrate electrodes most commonly used in electrochemical studies of long-range OGN ET/HT, and would be disturbed by electrode or solvent oxidation. Complex molecules are also subject to potential dependent surface reorganization reflected in capacitive signals that can resemble Faradaic signals ${ }^{36,37}$. Such signals can also show weak distance dependence and high sensitivity to base pair mismatch $^{37}$. Polaron conductivity ${ }^{29}$ is an attractive notion, as much fewer "hops" are needed, but electrochemical polaron signals have not been identified either. In the following we consider first recent single-molecule electrochemical in situ studies of duplex OGNs in Section 2, followed by a discussion of monolayer and single-molecule electrochemical behaviour of simple representatives of non-canonical G-quadruplexes in Section 3.

\section{In situ STM imaging and conductivity of pure and redox marked duplex OGNs 2.1 In situ STM imaging}

We consider first single-molecule in situ STM of single- and duplex-strand (ss and ds) OGNs. As for 
single-molecule protein studies, in situ STM of DNA/OGNs holds other challenges than imaging in air/UHV ${ }^{2,15,25,27}$. Solvation favours upright orientation, at potentials negative of the potential of zero charge, while target OGN molecules in air/UHV virtually always lie down on the substrate surfaces. In situ STM resolution of OGN in aqueous media is, however, lower than in UHV and has only recently reached the single-molecule level ${ }^{12,13,27}$. Oshiro and Maeda recorded single-molecule in situ STM of 11 bp OGNs that testify to upright OGN orientation and notably, to different STM contrasts for ss and ds OGNs, Fig.2A. ${ }^{12}$ Mechanistic implications were not, however, elaborated. Methylation of single-strand DNA on the Au electrode caused the conductivity of an 8 base pair OGN to decrease, enabling again ss and ds molecular scale distinction, Fig. 2B (step 3). ${ }^{13}$ Hansen, Salvatore, and associates used a different approach. They reported in situ STM of 13-20 bp duplex OGNs attached to $\mathrm{Au}(111)$-surfaces by a thiol linker. ${ }^{25,27}$ Targets were normal and "unlocked" DNA (UNA), functionalized by terpyridine linked $\mathrm{Ru}^{3+/ 2+}, \mathrm{Os}^{3+/ 2+}$ and $\mathrm{Fe}^{3+/ 2+}$ (Fig. 2C-D) ${ }^{25}$ Single-molecule resolution was achieved, with significantly stronger contrasts of redox marked than of unmarked OGNs, Fig.2E reflecting different single-molecule ET/HT mechanisms, cf. below. ${ }^{25,27}$

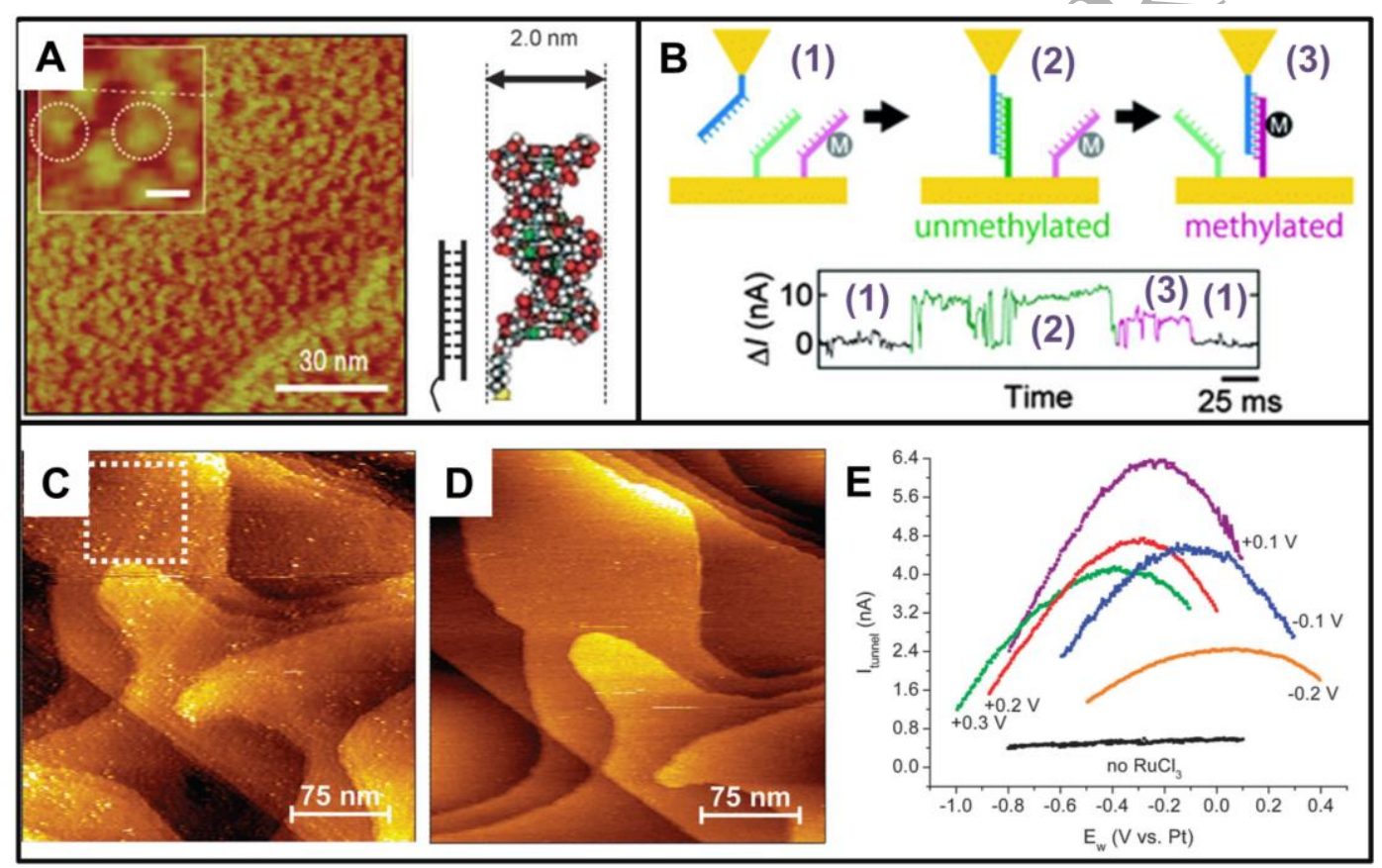

Fig. 2 (A) In situ STM image of 11-base ds OGN on Au-electrode surface (10 mM phosphate, $\mathrm{pH}$ 7.3). $E_{\text {sample }}=-0.2 \mathrm{~V}, E_{\text {bias }}=0.2 \mathrm{~V}$ (sample negative), $I_{\text {tunneling }}=200 \mathrm{pA}$. (B) Tunnelling current change of double-strand DNA by methylation in $0.1 \mathrm{M} \mathrm{NaClO}_{4}$. (C and D) In situ STM images of 15-base terpy-derivatized ds-UNA exposed to $\mathrm{Ru}(\mathrm{III}) . E_{\text {bias }}=0.2 \mathrm{~V}$. $E_{\text {sample }}=-0.2 \mathrm{~V}(\mathrm{C})$ and $-0.6 \mathrm{~V}(\mathrm{D})$. (E) $I_{\text {tunneling }}$ vs. $E_{\text {sample }}$ at fixed $E_{\text {bias }} .10 \mathrm{mM}$ phosphate, $\mathrm{pH}$ 7.0). From Refs. ${ }^{12,13,27}$ with permission.

\subsection{In situ electrochemical single-molecule conductivity of pure and redox marked duplex OGNs}

In situ single-molecule DNA/OGN studies have focused on conductivity rather than imaging. Singlemolecule visualization is appealing, but conductivity a more direct representation of biological single-molecule action, even though conductivity and STM/cAFM imaging are physically closely related. Tao pioneered in situ STM of redox molecules ${ }^{38}$. In many recent reports, using STM (B-J) techniques supported by quantum chemical computations and transport calculations ${ }^{39}$, Tao and associates have mapped electronic conductivity of ss and ds OGNs of variable length and composition. These studies, followed by studies by Diéz-Pérez, Hihath, and associates ${ }^{10,11,15,40,41}$ offer recent examples. The high data accuracy offers insight at several levels. Exponential distance decay was, for example observed but the decay factors, $\beta_{\text {eff }}$ assume values ranging from 0.6-0.7 $\AA^{-1}$ characteristic of tunnelling (superexchange) to much smaller values, characteristic of hopping or gating, cf. discussion in Section 1. The distance variation thus depends sensitively on base 
composition, stacking, and other structural details. Periodic oscillations in the conductivity/distance correlation ${ }^{11}$ were other observations. The parameters $\Delta \varepsilon$ and $\gamma$ in the coarse-grained distance relation eq.(2) in fact already depend on the distance and base composition, itself a source of oscillatory behaviour, particularly for small values of the $\gamma / \Delta \varepsilon$ ratio. The oscillatory behaviour was framed by the Büttiker theory of "partially coherent" charge transfer with "incoherent corrections" ${ }^{39}$. In other terms such notions relate to vibrationally activated multi-electron transfer in molecular junctions that prevails for strong electronic coupling of the target molecule to the enclosing electrodes ${ }^{2,8,34,35}$. The multifarious single-molecule electrochemical OGN conductivity behaviour thus supports base pair specific behaviour further sensitive to temperature and electrolyte composition.

In several studies, Kawai, Ohshiro and associates, Nishino and associates, and Majima and associates reported single-molecule in situ conductivity of pure and chemically modified OGNs ${ }^{22-24}$. Core notions were functionalized probing tips, and tunnelling current-distance and current-time recording as introduced by Nichols, Haiss, and associates ${ }^{42}$. The data included single-molecule mapping of ss/ds hybridization, and mismatch (polymorphism) detection. Together with the comprehensive studies of Tao and associates, these recent results have further lifted single-molecule DNA science. Transition metal binding to immobilized OGNs offers other current/overpotential/bias voltage STS, with strong signals particularly from Ru-bound OGNs ${ }^{27}$. The STS correlations showed systematically the multiphonon two-step hopping STS characteristics, Fig.2E, based on the views represented by eqs.(1) and (2) and by Fig.1B, lower left, with spectral features around the equilibrium potential but broadened by Ru-binding to multiple binding sites. Additional "hopping" conductivity channels along the bound $\mathrm{Ru}$-sites are therefore envisaged.

\section{Electrochemistry, in situ STM imaging and conductivity of DNA G-quadruplexes}

\subsection{Why electrochemical single-molecule approaches? G-quadruplex structure and the DNAzyme}

DNA strands assemble into triplex and quadruplex structures. Interest in the latter started with the discovery of hundreds of six-nucleotide single strand G-rich tandem repeats [(TTAGGG) $)_{n}$ ] forming "non-canonical" Hoogsteen quartets terminating the duplex DNA and protecting eukaryotic chromosome maintenance (Fig.3A and B) ${ }^{43-46}$ The quadruplexes, are denoted "telomeres" (Gr.: telos $=$ end; meros $=$ part $)$. They are conjectured to hold keys to age and cancer ${ }^{47-54}$ and stabilized by alkali metal ions and divalent cations $\left(\mathrm{Pb}^{2+}, \mathrm{Sr}^{2+}\right) .^{55-57}$ Porphyrins can stack onto the terminal G-quartet planes by л-л interactions as a telomerase inhibitor. ${ }^{58,59}$ G-quartet bound porphyrins also exhibit peroxidase activity and ultra-sensitivity to $\mathrm{K}^{+}, \mathrm{Na}^{+}$and $\mathrm{Pb}^{2+}$. All this has prompted the notion "DNAzyme" as a biomimetic enzyme (Fig. 3C). ${ }^{60-61}$ Novel electrochemical approaches to DNAzyme structure and enzyme dynamics based on single-molecule conductivity are therefore warranted, as for duplex OGNs and recently for the copper enzymes nitrite reductase ${ }^{62}$ and laccase ${ }^{63}$.

\subsection{A G-quadruplexes as molecular electronic conductors}

As for duplex DNA, the attractive looking three-dimensional (3D) variable-length G-quadruplexes have prompted views on G-quadruplexes in molecular electronics ${ }^{65}$. Similar to "smart" transition metal complexes ${ }^{1,2,14,66}$, metalloproteins ${ }^{2,6-8}$, and redox marked duplex $\mathrm{OGNs}^{25,27}$, single-molecule rectification, amplification etc. can be expected, provided that G-quadruplexes are good singlemolecule conductors, as expected by the electronically delocalized 4G-base quartets and strong Л-л electronic interactions among the G-quartets.

\subsubsection{Electrochemistry of G-quadruplex structures}

Photo-induced G-quadruplex conductivity in aqueous media is established ${ }^{68-70}$, but electrochemical studies of G-quadruplexes are very few. Doneaux and associates reported voltammetric and spectroscopic studies of $\left[\mathrm{Ru}\left(\mathrm{NH}_{3}\right)_{6}\right]^{3+}$ on polycrystalline gold surfaces modified by G-quadruplexes and G-duplex monolayers. ${ }^{67}\left[\mathrm{Ru}\left(\mathrm{NH}_{3}\right)_{6}\right]^{3+}$ binds selectively to G-quadruplex in the adlayers by nonelectrostatic interactions giving a new reduction peak at $-0.14 \mathrm{~V}$ (vs. SCE) in successive ac voltammograms. ${ }^{67}$ Apart from electroanalytical perspectives, this could point to G-quadruplex 
hopping via bound $\left[\mathrm{Ru}\left(\mathrm{NH}_{3}\right)_{6}\right]^{3+}$, as for Ru-complex binding to ds-OGNs, Section 2.

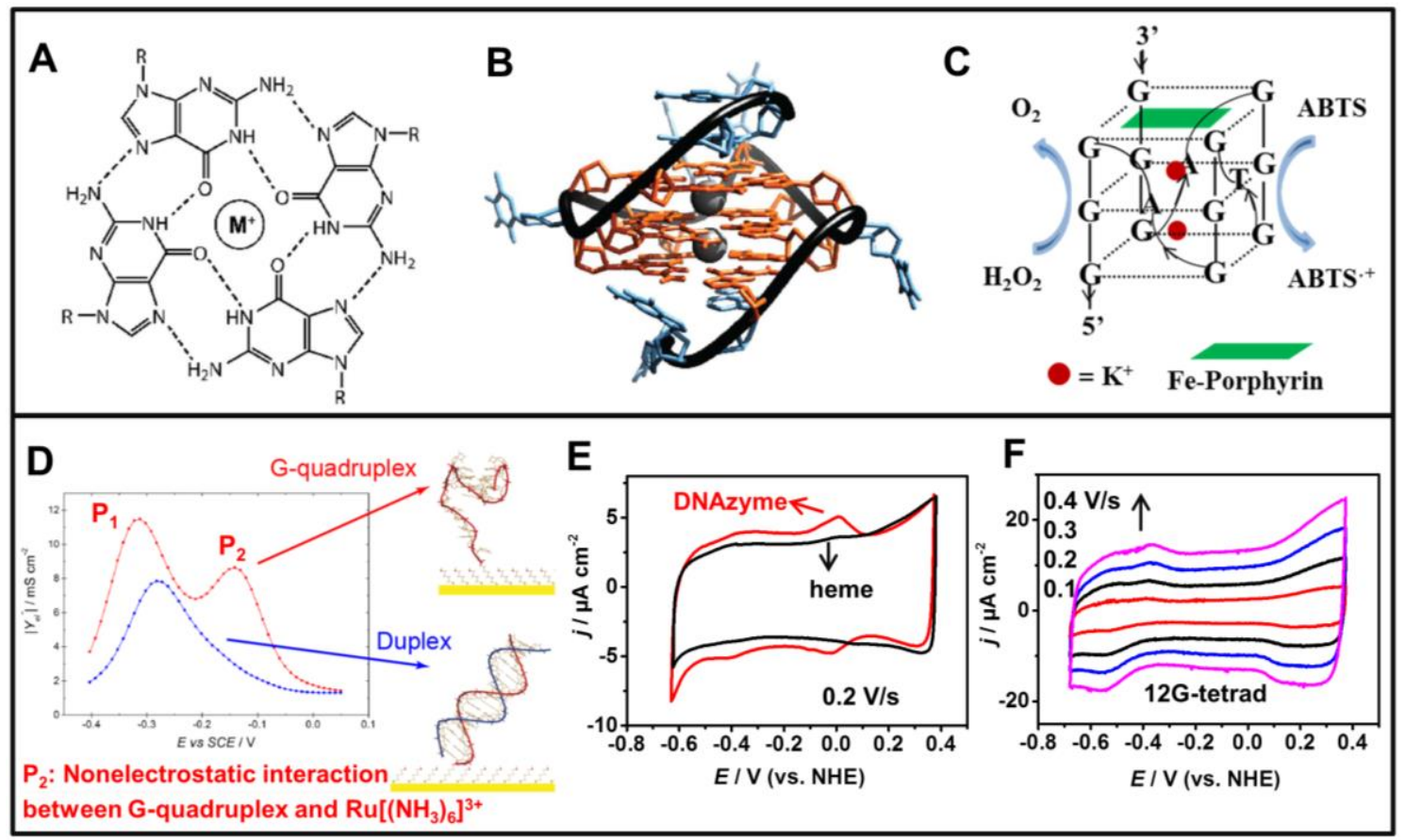

Fig. 3 (A) G-quartet structure coordinated with metal cations. (B) Solution NMR structure of c-myc Pu22 sequence 5'-TGAGGGTGGGTAGGGTGGGTAA-3' (PDB ID: 1XAV). From Ref. ${ }^{44}$ with permission. (C) DNAzyme of G-quadruplex with iron porphyrin IX (FePPIX, or heme). From Ref. ${ }^{64}$ with permission. (D) Successive ac voltammograms at G-quadruplex and duplex DNA modified electrodes. $10 \mathrm{mM}$ Tris buffer $\left(\mathrm{pH}\right.$ 7.4) $+100 \mu \mathrm{M}\left[\mathrm{Ru}\left(\mathrm{NH}_{3}\right)_{6}\right]^{3+}$. One voltammogram plotted every two measurements. $f=37 \mathrm{~Hz}, \mathrm{~T}=20{ }^{\circ} \mathrm{C}$. From Ref. ${ }^{67}$ with permission. (E and $\mathrm{F}$ ) CVs of $12 \mathrm{G}-$ quadruplex based DNAzyme and free heme (E) and of FePPIX free 12G-quadruplex (F) on $\mathrm{Au}(111)-$ electrode in $5 \mathrm{mM}$ sodium acetate, $\mathrm{pH}$ 7.2. From Ref. ${ }^{64}$ with permission.

Carbon materials enable following electrochemical guanine hole generation. Olivera-Brett and associates reported recently a voltammetric and AFM study of the time evolution of $\mathrm{G}_{10}$, $\mathrm{TG}_{9}$ and $\mathrm{TG}_{8} \mathrm{~T}$ on glassy carbon (voltammetry) and highly oriented pyrolytic graphite $(\mathrm{AFM})^{71}$ from single strands to folded G-quadruplexes. AFM (in air) disclosed morphologically different aggregates in the adlayers. Molecular resolution was not achieved, and the AFM air environment poses recognized limitations, but use of carbon might offer promise for high-resolution mapping of electron or hole creation, once binding in welldefined orientations on the electrode surfaces can be controlled.

Zhang and associates recently reported a combined electrochemical and in situ STM study of the smallest, 12G-containing (5'-SH- $\left(\mathrm{CH}_{2}\right)_{6}$-GGG A GGGT GGGA GGG) G-quadruplex FePPIX DNAzyme, on $\mathrm{Au}(111)$-electrode surfaces, Fig. 3C ${ }^{64}$. Fig. 3E shows cyclic voltammograms (CVs) of DNAzyme with pure FePPIX (heme) modified Au(111)-electrode as a reference. A pair of voltammetric peaks at $0.0 \mathrm{~V}$ (NHE) correspond to a dense monolayer, notably with a large electrochemical rate constant $(6.0 \pm 0.4) \mathrm{s}^{-1}$. The peak potential is significantly higher than for FePPIX on graphite $(\approx-0.2 \mathrm{~V}$ vs NHE) due to specific FePPIX/G-quartet interactions. The large rate constant suggests facile ET/HT through the G-quadruplex, but this is not conclusive, as the FePPIX binding end of the G-quadruplex is presently unknown, cf. further Section 3.2.2. Another pair of peaks around $-0.4 \mathrm{~V}$ for both DNAzyme and FePPIX free G-quadruplex was assigned a capacitive origin as for the reorientational capacitive peak reported for duplex OGNs, Fig. 3F. ${ }^{37}$ 


\subsubsection{Hopping and G-quadruplex conductivity}

Variable-length G-quadruplexes form differently stacked conformations, with strong $\pi-\pi$ couplings ${ }^{68}$. In comparison to duplex OGNs photo-induced ET/HT in solution, there are much fewer reports of Gquadruplex conductivity ${ }^{24,65,69,70}$. These studies point to higher conductivity due to the larger number of $\pi$-contacts and more delocalized electronic states, but the same issues are left as for G-duplex electrochemical conductivity, i.e. how the excess charges are created electrochemically in the first place, and why voltammetric ET/HT signals are not apparent.

Theoretical studies have addressed the coupling between differently stacked G-bases ${ }^{68,71}$. The couplings are strong, with $T_{\mathrm{DA}} \approx 0.03-0.3 \mathrm{eV}$. Such values imply that individual hops would be in the adiabatic limit, $\kappa \rightarrow 1$, eq.(1) ${ }^{33}$ and tunnelling only reflected in lowered activation free energy, $\Delta G^{ \pm}$ $\rightarrow \Delta G^{\neq}-1 / 2 T_{\mathrm{DA}}$. With reorganization free energies of $0.5-1 \mathrm{eV}$ the activation free energy can therefore almost vanish for favourably stacked G-quartets and hopping be an efficient photo-conductivity hopping mechanism, eqs.(1) and (2), once the excess electrons or holes have been created.

\subsubsection{Single-molecule G-quadruplex conductivity - STM, cAFM and in situ STM}

Following comprehensive studies of duplex OGNs, Porath and associates reported single-molecule mapping of long G-quadruplexes immobilized laterally on mica surfaces, using STM and cAFM ${ }^{65,72}$. Sub-molecular image resolution was achieved and single-molecule conductivity mapped, albeit in non-biological (vacuum) environments. Weak conductivity distance dependence over $>100 \mathrm{~nm}$ could be mapped by positioning the cAFM tip along the strands, with current observed at several volts threshold voltages, strongly indicative of "hopping" conductivity. These discoveries are important, but air/vacuum and high voltages leave issues regarding solvation, counter ions, biomolecules under external stress etc. compared with natural biological environments. Such questions can be approached differently, by electrochemical STM (or cAFM) and have been addressed recently ${ }^{64}$. In situ STM to molecular resolution of the 5'GGG A GGGT GGGA GGG 3 G4-quadruplex DNAzyme in biological buffer under electrochemical control was achieved, cf. Section 3.2.1, disclosing different surface structures. Domain 1, Fig.4 shows both quadratic molecular scale and larger octagonal entities (Fig.4C). The former resemble the 12G-quadruplex box. The larger octagonal units could then be water networks stretching out from the quartets, Fig. 4D. Similar quartet structures appear in UHV Gassemblies on $\mathrm{Au}(111)$ surfaces. ${ }^{73}$ The larger side length, $1.6 \pm 0.2 \mathrm{~nm}$ of the in situ 12G-quadruplex than for the UHV G-quartet $(0.67 \mathrm{~mm})$ again most likely discloses water networks. Domain 2 shows linear arrays, perhaps unfolded single strands. Even though voltammetrically robust, surface bound G-quadruplexes thus appear to be mobile molecular units. In this respect they follow duplex OGNs.

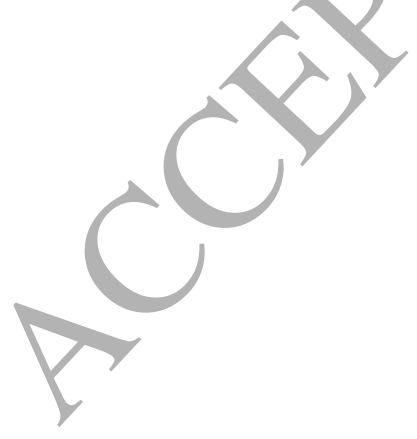



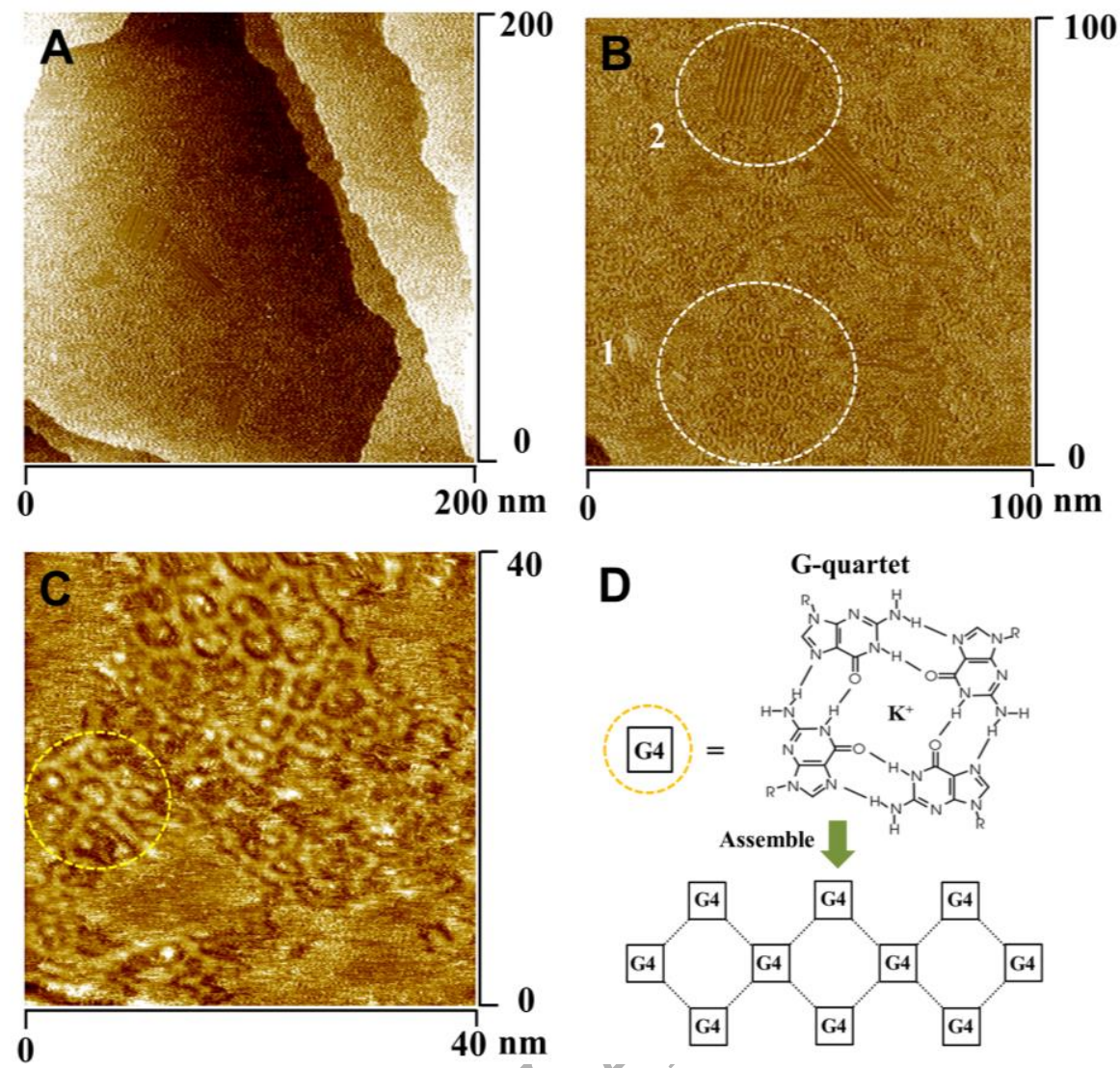

Fig. 4 (A-C) In situ STM images of DNAzyme on Au(111)-electrode surface. $5 \mathrm{mM}$ sodium acetate, $\mathrm{pH}$ 7.0. $E_{\text {sample }}=0.49 \mathrm{~V}$ (vs. NHE), $E_{\text {bias }}=-0.48 \mathrm{~V}$. $I_{\text {tunneling }}=0.09 \mathrm{nA}$. (D) Schematic structure of the G- and octagonal G-tetrad superstructures. From Ref. ${ }^{64}$ with permission.

\section{Concluding remarks}

We have provided a discussion of single-molecule in situ imaging and conductivity of duplex and quadruplex DNA/OGNs with emphasis on electrochemistry on single-crystal $\mathrm{Au}(111)$-electrode surfaces and in situ STM. The mechanistic challenges regarding OGN conduction in air/UHV and aqueous media noted for duplex OGNs apply as well to quadruplex OGNs, for which, however, only few electrochemical and in situ single-molecule studies are reported. With a view on the success of B-J techniques for single-molecule in situ duplex OGN conductivity, this technology is likely to hold promise also for systematic single-molecule, variable-length G-quadruplex conductivity. In view of the strong $\pi-\pi$ interactions between the G-quartets such studies could also open paths to the subtle region between strongly adiabatic hopping and superexchange/tunnelling in OGN charge transport. The outcomes of such studies could also offer guidance to new types of G-quadruplex sensors and possible forthcoming molecular scale electronics.

\section{Acknowledgement}

LZ is grateful for the award of a H.C. Ørsted Marie Curie CoFund postdoctoral fellowship. Financial support from The Danish Council for Independent Research for YDUN project (DFF 4093 00297), the Lundbeck Foundation (R141-2013-13273), and the Russian Science Foundation (17-13-01274) is acknowledged. We wish to thank Professor Jesper Wengel, the Nucleic Acid Center, University of Southern Denmark for helpful discussions.

\section{References}

(1) Nichols, R. J.; Higgins, S. J.: Single Molecule Nanoelectrochemistry in Electrical Junctions. Acc. 
Chem. Res. 2016, 49, 2640-2648.

- Detailed and updated overview of electrochemistry and in situ scanning tunnelling microscopy of redox molecules at the single-molecule level

(2) Zhang, J.; Kuznetsov, A. M.; Medvedev, I. G.; Chi, Q.; Albrecht, T.; Jensen, P. S.; Ulstrup, J.: Single-Molecule Electron Transfer in Electrochemical Environments. Chem. Rev. 2008, 108, 2737-2791.

- Detailed overview of electrochemical electron transfer and in situ scanning tunnelling microscopy imaging of single molecules and biomolecules.

(3) Friis, E. P.; Andersen, J. E. T.; Kharkats, Y. I.; Kuznetsov, A. M.; Nichols, R. J.; Zhang, J.-D.; Ulstrup, J.: An approach to long-range electron transfer mechanisms in metalloproteins: In situ scanning tunneling microscopy with submolecular resolution. Proc. Natl. Acad. Sci. 1999, 96, 1379-1384.

(4) Alessandrini, A.; Gerunda, M.; Canters, G. W.; Verbeet, M. P.; Facci, P.: Electron tunnelling through azurin is mediated by the active site $\mathrm{Cu}$ ion. Chem. Phys. Lett. 2003, 376, 625-630.

(5) Chi, Q.; Farver, O.; Ulstrup, J.: Long-range protein electron transfer observed at the singlemolecule level: In situ mapping of redox-gated tunneling resonance. Proc. Natl. Acad. Sci 2005, 102, 16203-16208.

(6) Alessandrini, A.; Facci, P.: Electron transfer in nanobiodevices. Eur. Polym. J. 2016, 83, 450-466.

(7) Baldacchini, C.; Bizzarri, A. R.; Cannistraro, S.: Electron transfer, conduction and biorecognition properties of the redox metalloprotein Azurin assembled onto inorganic substrates. Eur. Polym. J. 2016, 83, 407-427.

(8) Della Pia, E. A.; Chi, Q.; Macdonald, J. E.; Ulstrup, J.; Jones, D. D.; Elliott, M.: Fast electron transfer through a single molecule natively structured redox protein. Nanoscale 2012, 4, 71067113.

(9) Hao, X.; Zhang, J.; Christensen, H. E. M.; Wang, H.; Ulstrup, J.: Electrochemical SingleMolecule AFM of the Redox Metalloenzyme Copper Nitrite Reductase in Action. Chemphyschem 2012, 13, 2919-2924.

(10) Hihath, J.; Xu, B.; Zhang, P.; Tao, N.: Study of single-nucleotide polymorphisms by means of electrical conductance measurements. Proc. Natl. Acad. Sci. 2005, 102, 16979-16983.

(11) Xiang, L.; Palma, J. L.; Bruot, C.; Mujica, V.; Ratner, M. A.; Tao, N.: Intermediate tunnellinghopping regime in DNA charge, transport. Nat. Chem. 2015, 7, 221-226.

- High-resolution break-junction study of distance dependent single-molecule conductivity of DNA oligonucleotides in solution disclosing subtleties of coherent and incoherent transport.

(12) Ohshiro, T.; Maeda, M.: Single-molecule imaging of DNA duplexes immobilized on surfaces with a scanning tunneling microscope. Chem. Commun. 2010, 46, 2581-2583.

- First single-molecule single-strand and duplex STM imaging in solution.

(13) Bui, P. T.; Nishino, T.; Shiigi, H.; Nagaoka, T.: One-by-one single-molecule detection of mutated nucleobases by monitoring tunneling current using a DNA tip. Chem. Commun. 2015, 51, 1666-1669.

(14) Káwai, K.; Majima, T.: Hole Transfer Kinetics of DNA. Acc. Chem. Res. 2013, 46, 2616-2625.

(15) Artés, J. M.; Li, Y.; Qi, J.; Anantram, M. P.; Hihath, J.: Conformational gating of DNA conductance. Nat. Commun. 2015, 6, 8870.

(16) Ryndyk, D. A.; Shapir, E.; Porath, D.; Calzolari, A.; Di Felice, R.; Cuniberti, G.: Scanning Tunneling Spectroscopy of Single DNA Molecules. ACS Nano 2009, 3, 1651-1656.

(17) Genereux, J. C.; Barton, J. K.: Mechanisms for DNA Charge Transport. Chem. Rev. 2010, 110, 1642-1662.

- - Detailed overview of mechanisms of photo-induced and electrochemical charge transfer of pure and modified DNA based molecules. 
(18) Arnold, Anna R.; Grodick, Michael A.; Barton, Jacqueline K.: DNA Charge Transport: from Chemical Principles to the Cell. Cell Chem. Biol. 2016, 23, 183-197.

(19) Sontz, P. A.; Muren, N. B.; Barton, J. K.: DNA Charge Transport for Sensing and Signaling. Acc. Chem. Res. 2012, 45, 1792-1800.

(20) Jortner, J.; Bixon, M.; Langenbacher, T.; Michel-Beyerle, M. E.: Charge transfer and transport in DNA. Proc. Natl. Acad. Sci. 1998, 95, 12759-12765.

- - Introduction and first transparent theoretical frame for the hopping mechanism of electronic conductivity of DNA based molecules.

(21) Giese, B.; Amaudrut, J.; Kohler, A.-K.; Spormann, M.; Wessely, S.: Direct observation of hole transfer through DNA by hopping between adenine bases and by tunnelling. Nature 2001, 412, 318-320.

(22) Ohshiro, T.; Matsubara, K.; Tsutsui, M.; Furuhashi, M.; Taniguchi, M.; Kawai, T.: SingleMolecule Electrical Random Resequencing of DNA and RNA. Sci. Rep, 2012, 2, 501.

(23) Matsuura, H.; Hokonohara, H.; Sugita, T.; Takagi, A.; Suzuki, K.; Matsumoto, T.; Kawai, T.: DNA observation with scanning tunneling microscope using a solution. J. Appl. Phys. 2011, 109.

(24) Choi, J.; Park, J.; Tanaka, A.; Park, M. J.; Jang, Y. J.; Fujitsuka, M.; Kim, S. K.; Majima, T.: Hole Trapping of G-Quartets in a G-Quadruplex. Angew. Chem. Int. Ed. 2013, 52, 1134-1138.

- Hole conduction in a G-quadruplex molecule.

(25) Salvatore, P.; Zeng, D.; Karlsen, K. K.; Chi, Q.; Wengel, J.; Ulstrup, J.: Electrochemistry of Single Metalloprotein and DNA-Based Molecules at $\mathrm{Au}(111)$ Electrode Surfaces. Chemphyschem 2013, 14, 2101-2111.

(26) Wong, E. L. S.; Gooding, J. J.: Charge Transfer through DNA: A Selective Electrochemical DNA Biosensor. Anal. Chem. 2006, 78, 2138-2144.

(27) Hansen, A. G.; Salvatore, P.; Karlsen, K. K.; Nichols, R. J.; Wengel, J.; Ulstrup, J.: Electrochemistry and in situ scanning tunnelling microscopy of pure and redox-marked DNAand UNA-based oligonucleotides on Au(111)-electrode surfaces. Phys. Chem. Chem. Phys. 2013, $15,776-786$.

(28) Gorodetsky, A. A.; Buzzeo, M. C.; Barton, J. K.: DNA-Mediated Electrochemistry. Bioconjugate Chem. 2008, 19, 2285-2296.

(29) Conwell, E. M.: Charge transport in DNA in solution: The role of polarons. Proc. Natl. Acad. Sci. 2005, 102, 8795-8799.

(30) Dogonadze, R. R.; Ulstrup, J.; Kharkats, Y. I.: A theory of electrode reactions through bridge transition states; bridges with a discrete electronic spectrum. J. Electroanal. Chem. Interfacial Electrochem. 1972, 39, 47-61.

(31) Kuznetsov, A. M.; Ulstrup, J.: Dissipative relaxation of a low-energy intermediate electronic state in three-level electron transfer. Chem. Phys. 1991, 157, 25-33.

(32) Kuznetsov, A. M.; Sommer-Larsen, P.; Ulstrup, J.: Resonance and environmental fluctuation effects in STM currents through large adsorbed molecules. Surf. Sci. 1992, 275, 52-64.

(33) Kuznetsov, A. M.; Ulstrup, J: Electron Transfer in Chemistry and Biology: An Introduction to the Theory. Wiley, Chichester 1998.

(34) Kuznetsov, A. M.; Ulstrup, J.: Mechanisms of in Situ Scanning Tunnelling Microscopy of Organized Redox Molecular Assemblies. J. Phys.Chem. A 2000, 104, 11531-11540. Errata: 2001, 105, 7494-7494.

(35) Zhang, J.; Chi, Q.; Kuznetsov, A. M.; Hansen, A. G.; Wackerbarth, H.; Christensen, H. E. M.; Andersen, J. E. T.; Ulstrup, J.: Electronic Properties of Functional Biomolecules at Metal/Aqueous Solution Interfaces. J. Phys. Chem. B 2002, 106, 1131-1152.

(36) Zhang, J. D.; Demetriou, A.; Welinder, A. C.; Albrecht, T.; Nichols, R. J.; Ulstrup, J.: Potentialinduced structural transitions of DL-homocysteine monolayers on $\mathrm{Au}(111)$ electrode surfaces. Chem. Phys. 2005, 319, 210-221. 
(37) Salvatore, P.; Karlsen, K. K.; Hansen, A. G.; Zhang, J.; Nichols, R. J.; Ulstrup, J.: Polycation Induced Potential Dependent Structural Transitions of Oligonucleotide Monolayers on $\mathrm{Au}(111)$ Surfaces. J. Am. Chem. Soc. 2012, 134, 19092-19098.

(38) Tao, N. J.: Probing Potential-Tuned Resonant Tunneling through Redox Molecules with Scanning Tunneling Microscopy. Phys. Rev. Lett. 1996, 76, 4066-4069.

- - First report of electrochemical single-molecule tunnelling/overpotential spectroscopy.

(39) Büttiker, M.: Coherent and sequential tunneling in series barriers. IBM J. Res. Dev. 1988, 32, 63-75.

(40) Xiang, L.; Palma, J. L.; Li, Y.; Mujica, V.; Ratner, M. A.; Tao, N.: Gate-controlled conductance switching in DNA. Nat. Commun. 2017, 8, 14471.

(41) Artés, J. M.; López-Martínez, M.; Díez-Pérez, I.; Sanz, F.; Gorostiza, P.: Nanoscale charge transfer in redox proteins and DNA: Towards biomolecular electronics. Electrochimica Acta 2014, 140, 83-95.

(42) Haiss, W.; Nichols, R.J.; van Zalinge, H.; Higgins, Bethell, D.; Schiffrin, D.J.: Measurement of single molecule conductivity using the spontaneous formation of molecular wires. Phys. Chem. Chem. Phys. 2004, 6, 4330-4337.

(43) Huppert, J. L.: Four-stranded DNA: cancer, gene regulation and drug development. Philos. Trans. A Math. Phys. Eng. Sci. 2007, 365, 2969-2984.

(44) Harkness, R. W.; Mittermaier, A. K.: G-quadruplex dynamics. Biochim. Biophys. Acta, Proteins Proteomics 2017.

(45) Zijlmans, J. M. J. M.; Martens, U. M.; Poon, S. S. S.; Raap, A. K.; Tanke, H. J.; Ward, R. K.; Lansdorp, P. M.: Telomeres in the mouse have large inter-chromosomal variations in the number of T2AG3 repeats. Proc. Natl. Acad. Sci. 1997, 94, 7423-7428.

(46) Hodes, R. J.; Hathcock, K. S.; Weng, N.-p.: Telomeres in T and B cells. Nat. Rev. Immunol. 2002, 2, 699-706.

(47) Wright, W. E.; Tesmer, V. M.; Huffman, K. E.; Levene, S. D.; Shay, J. W.: Normal human chromosomes have long G-rich telomeric overhangs at one end. Genes Dev. 1997, 11, 28012809.

(48) Rhodes, D.; Lipps, H. J.: G-quadruplexes and their regulatory roles in biology. Nucleic Acids Res. 2015, 43, 8627-8637.

(49) Blackburn, E. H.: Telomeres- No end in sight. Cell 1994, 77, 621-623.

(50) Feng, J.; Funk, W.; Wang, S.; Weinrich, S.; Avilion, A.; Chiu, C.; Adams, R.; Chang, E.; Allsopp, R.; Yu, J.; al., e.: The RNA component of human telomerase. Science 1995, 269, 12361241.

(51) Wang, Q.; Liu, J.-q.; Chen, Z.; Zheng, K.-w.; Chen, C.-Y.; Hao, Y.Hh.; Tan, Z.: G-quadruplex formation at the $3^{\prime}$ end of telomere DNA inhibits its extension by telomerase, polymerase and unwinding by helicase. Nucleic Acids Res. 2011, 39, 6229-6237.

(52) Shay, J. W.; Bacchetti, S.: A survey of telomerase activity in human cancer. European Journal of Cancer 1997, 33, 787-791.

(53) Zahler, A. M.; Williamson, J. R.; Cech, T. R.; Prescott, D. M.: Inhibition of elomerase by Gquartet DNA structures. Nature 1991, 350, 718-720.

(54) Street, S. T. G.; Chin, D. N.; Hollingworth, G. J.; Berry, M.; Morales, J. C.; Galan, M. C.: Divalent Naphthalene Diimide Ligands Display High Selectivity for the Human Telomeric Gquadruplex in $\mathrm{K}^{+}$Buffer. Chem. Eur. J. 2017, 23, 6953-6958.

(55) Howard, F. B.; Miles, H. T.: Poly(inosinic acid) helixes: essential chelation of alkali metal ions in the axial channel. Biochemistry 1982, 21, 6736-6745.

(56) Smirnov, I.; Shafer, R. H.: Lead is unusually effective in sequence-specific folding of DNA1. $J$. Mol. Biol. 2000, 296, 1-5.

(57) Li, T.; Wang, E.; Dong, S.: Potassium-Lead-Switched G-Quadruplexes: A New Class of DNA Logic Gates. J. Am. Chem. Soc. 2009, 131, 15082-15083.

(58) Wheelhouse, R. T.; Sun, D.; Han, H.; Han, F. X.; Hurley, L. H.: Cationic Porphyrins as 
Telomerase Inhibitors: the Interaction of Tetra-(N-methyl-4-pyridyl)porphine with Quadruplex DNA. J. Am. Chem. Soc. 1998, 120, 3261-3262.

(59) Neidle, S.: Quadruplex Nucleic Acids as Novel Therapeutic Targets. J. Med.Chem. 2016, 59, 5987-6011.

(60) Qin, H. X.; Ren, J. T.; Wang, J. H.; Luedtke, N. W.; Wang, E. K.: G-Quadruplex-Modulated Fluorescence Detection of Potassium in the Presence of a 3500-Fold Excess of Sodium Ions. Anal. Chem. 2010, 82, 8356-8360.

(61) Sun, H. J.; Li, X. H.; Li, Y. C.; Fan, L. Z.; Kraatz, H. B.: A novel colorimetric potassium sensor based on the substitution of lead from G-quadruplex. Analyst 2013, 138, 856-862.

(62) Zhang, J. D.; Chi, Q. J.; Hansen, A. G.; Jensen, P. S.; Salvatore, P.; Ulstrup, J.: Interfacial electrochemical electron transfer in biology - Towards the level of the single molecule. FEBS Lett. 2012, 586, 526-535.

(63) Climent, V.; Zhang, J. D.; Friis, E. P.; Ostergaard, L. H.; Ulstrup, J.: Voltammetry and SingleMolecule in Situ Scanning Tunneling Microscopy of Laccases and Bilirubin Oxidase in Electrocatalytic Dioxygen Reduction on Au(111) Single-Crystal Electrodes, J. Phys. Chem. C 2012, 116, 1232-1243.

(64) Zhang, L.; Ulstrup, J.; Zhang, J. D.: Voltammetry and molecular assembly of G-quadruplex DNAzyme on single-crystal $\mathrm{Au}(111)$-electrode surfaces - hemin as an electrochemical intercalator. Faraday Discuss. 2016, 193, 99-112.

(65) Shapir, E.; Sagiv, L.; Borovok, N.; Molotski, T.; Kotlyar, A. B.; Porath, D.: High-Resolution STM Imaging of Novel Single G4-DNA Molecules. J. Phys. Chem. B 2008, 112, 9267-9269.

(66) Albrecht, T.; Guckian, A.; Ulstrup, J.; Vos, J. G.: Transistor-like Behavior of Transition Metal Complexes. Nano Lett. 2005, 5, 1451-1455.

(67) De Rache, A.; Doneux, T.; Buess-Herman, C.: Electrochemical Discrimination between GQuadruplex and Duplex DNA. Anal. Chem. 2014, 86, 8057-8065.

(68) Lech, C. J.; Phan, A. T.; Michel-Beyerle, M.-E.; Voityuk, A. A.: Electron-Hole Transfer in GQuadruplexes with Different Tetrad Stacking Geometries: A Combined QM and MD Study. J. Phys. Chem. B 2013, 117, 9851-9856.

- First detailed theoretical study of structure/function correlations in the electronic conductivity of G-quadruplex molecules.

(69) Liu, S.-P.; Weisbrod, S.H.; Tang, Z., Marx, A.; Scheer, E.; Erbe, A.: Direct measurement of electrical transport through G-quadruplex DNA with mechanically controllable breakjunction electrodes. Angew. Chem. Int. Ed. 2010, 49, 3313-3316.

- First direct study of the conductivity of single Q-quadruplex molecules in solution.

(70) Liu, S.; Zhang, X; Luo, W.; Gue, X.; Steigerwald, M.L.; Fang, X.: Single-molecule detection of proteins using aptamer-functionalized molecular electronic devices. Angew. Chem. Int. Ed. 2011, 50, 2496-2502.

- Interaction between proteins and G-quadruplex structures ate the single-molecule level.

(71) Chiorcea-Paquim, A.; Santos, P.V.; Eritja, R.; Oliveira-Brett, A.M.: Self-assembled Gquadruplex nanostructures: AFM and voltammetric characterization. Phys. Chem. Chem. Phys. 2013, 15, 9117-9124.

- Important electrochemical study of the time evolution from single-strand to G-quadruplex.

(72) Livshits, G.L.; Sterne, A; Totem, D.; borovok, N.; Eidelshtein, G.; Migliore, A.; Penzo, E.; Wind, S.J.; Di Felice, R.; Skourtis, S.S.; Cuevas, J.C.; Gurevich, L.; Kotlyar, A.B.; Porath, D.: Long-range charge transport in single G-quadruplex DNA molecules. Nature Nanotechnol. 2014, 9, 1040-1046.

- - High-resolution ex situ imaging of variable-length G-quadruplexes to sub-molecular resolution and distance dependent current/bias voltage correlations of single quadruplex molecules. 
(73) Zhang, C.; Xie, L.; Wang, L.; Kong, H.; Tan, Q.; Xu, W.: Atomic-Scale Insight into Tautomeric Recognition, Separation, and Interconversion of Guanine Molecular Networks on Au(111). $J$. Am. Chem. Soc. 2015, 137, 11795-11800. 
TOC

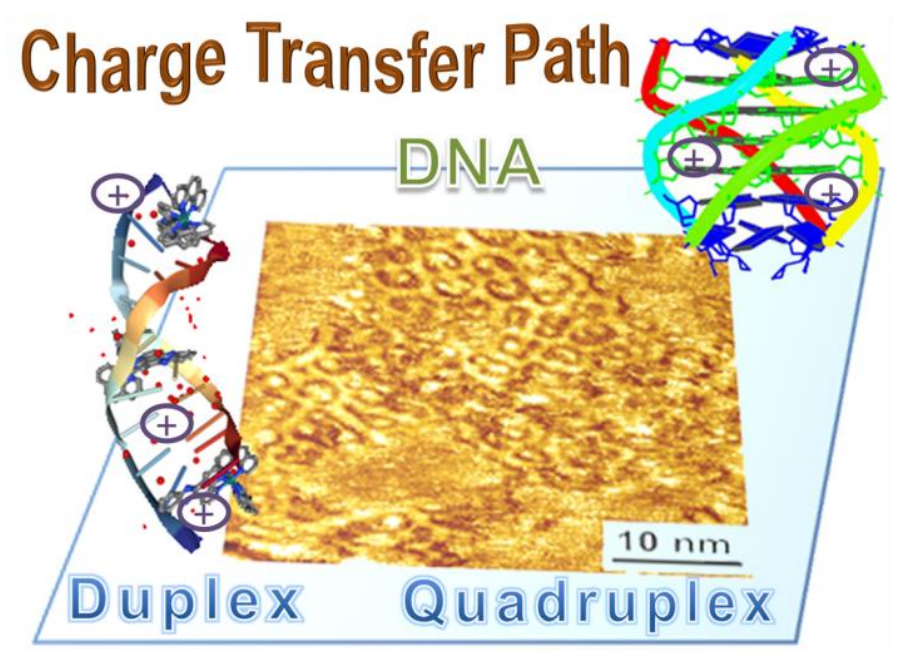

